\title{
A Co-evolution Framework towards Stable Designs from Radical Innovations for Organizations Using IT
}

\author{
Lakshminarayana Kompella
}

\begin{abstract}
The purpose of this paper is to theoretically and empirically explore how organizations can enable radical product innovations to cumulate as stable designs. Radical product innovations are organizational responses to external triggers that cause transitions. To manage in transitions, it is necessary for radical product innovations to cumulate as stable designs. Organizations ability to co-evolve with the environment does influence innovations to cumulate as stable designs; to examine this, the author selected public procurement that uses IT as radical product innovation with pronounced environmental influence, government's interventionist approach. The author used multiple case-study and obtained diverse analytic and heuristic views. From the cases, the author noted that actors did consider local and contingent factors only that resulted in certain radical innovations cumulating as stable designs. As an initial starting point, such actions are appropriate but organizational actions to expand their initial actions with a co-evolutionary framework that considers social contexts.
\end{abstract}

Keywords: socio-technical systems; radical innovations; open systems; co-evolution; case studies.

Submitted: March 27 $2019 /$ Approved: June $12^{\text {th }}, 2019$

\section{Introduction}

Organizations focus on supply and demand, often, with emphasis on rational decision-making. Along with supply and demand, utility and profit maximization also play a key role. Economic growth is not necessarily based on capital accumulation but also on innovation and knowledge. Both neoclassical and innovative economics focus on optimized utilization of resources while innovative economics emphasizes responses to change. The supply and demand and, in certain geographies, governments interventionist role also decide the organization's responses to change. Organizations respond to change with radical and incremental innovation. Frameworks such as Multi-level perspective (MLP) defined the origin of both radical and incremental innovations. The latter occurs in the regime level of MLP while the radical innovations occur at the niche level. The external and internal factors influence innovations both at the regime and niche level. The external factors such as interventionist approach, supply-push and demand-pull and internal factors such as profit maximization, knowledge accumulation and so forth decide the extent to which regime supports innovation including the adoption of radical innovations.

Organizations deal with socio-technical systems characterized by heterogeneous configurations such as policy, technology, regulatory, user-preferences and so forth. These configurations also decide the extent to which adoption of innovation occur at the regime and niche level actors' responses to external triggers with radical innovations. The cumulation of radical innovations as stable designs at regime level signals the adoption of radical innovations at the regime level. For radical innovations to cumulate as stable designs, apart from technological advances the actors need to change the configurations by considering external and internal factors. The internal factors manifest as 1) problems with the existing technology, 2) multiple actors' acceptance of radical innovation, 3) integrating radical innovation into the socio-technical system architecture with appropriate changes to radical process innovations 4 ) ease at which changes to organizational culture occur to assimilate both product and process innovations and finally, 5) organizations receptiveness to environmental factors and environment around it. The interoperations, ruleset based interactions, among the stakeholders do compound the internal factors. Moreover, based on the domain of the socio-technical system rule-sets do vary.

To examine the cumulation of radical innovations as stable designs, the author selected organizations that have a combination of interventionist approach, latest technology such as IT, supply-push and demand-pull characteristics, and regime actors who have entrenched certain rule-sets over a long period. Therefore, the author selected organizations from Central and state government of India. Moreover, organizations from Central and state governments, for a better response to the external and internal factors, did IT-enable their procurement activities. By examining the procurement activities of government organizations, the author plans to get insights into their adoption of radical innovations. For a better display of supply-push and demand-pull, the author considered only procurement activities of these organizations. These organizations did IT enable their procurement activities. IT innovations address spatial dimension factors, for example, address physical factor by enabling actors to access information from anywhere in the world and various possibilities of search such as keywords, syntactic and semantic processing address knowledge sharing possibilities to address knowledge asymmetries. Indeed, IT-based systems improve interoperations between organizations. In the next subsection, the author discusses expansion possibilities with IT.

School of Management, National Institute of Technology Warangal, Warangal, Telangana, India. E-mail:Inkompella@gmail.com 


\section{Organization and IT}

IT innovations were initially used for automation of organizational activities. Both IT innovations and the organization's external triggers influenced and did get influenced by one another. Thereby, IT innovations such as higher processing, memory, storage, distributed systems with localized and centralized functions enabled organizations to move away from work within their organizational boundaries, and, start aligning their work to span multiple organizations. In other words, organizations focused on workflow or the process. However, unlike workflow, information remained within organizational boundaries and based on authentication, authorization, and policies decided the users' usage of resources (accounting). With the advent of IT innovations such as cloud computing, researchers such as Zammuto et al., (2007) content there is a possibility of organizing around information. Zammuto et al., (2007) feel that organizing around information new forms of interaction are possible, and, organizations can explore new forms of organizing. Zammuto et al., (2007) argue that though IT and organizations exist independently of each other having their own potentials and constraints, we require necessary theories to explain affordances that arise when IT and organizations enact together. Zammuto et al., (2007) identified five affordances 1) visualizing entire work process, 2) real-time/flexible products and services 3) mass collaboration 4) virtual collaboration and 5) simulation representation from the intersection of IT and organization. With these five affordances, Zammuto et al., (2007) want to inspire organization and information science scholars to open the black box of IT and organization for new forms of organizing.

To open the black box of IT and organizations, the view through which we view IT and organizations become important. The two predominant views are the lens of realism and constructivism. Realism views that worldly objects have inherent properties that act as constraints on observational accounts. In constructivism, the reality of objects is itself an outcome of discursive practices in relation to the object. Interlinking and intertwining of social processes and technology artifacts do occur and we require to draw conclusions from the ways in which they are. The characteristics of technology and their relationship with social structures result in both being socially constructed. Therefore, Hutchby (2001) states that anti-determinism takes the view of anti-essentialism meaning - opposition to the view that technological artifacts have any inherent properties outside the interpretive work which humans engage. Literature has references where some of the significant constructionist works fall into unwelcome realistic assumptions (Hutchby, 2001). For example, socio-technical interactionism by Mackenzie and Wajcman (1999) say that specific functions and social effects of technology are bound to social factors, resulting in explanations struggling with a dualism between technology and the society. Reconciling between the opposite poles of constructivism and realism, Hutchby (2001) states, 'it requires seeing technologies neither in terms of their interpretive textual properties nor of their essential technical properties, but in terms of their affordances and this third way opens the way for new analyses in identifying technological artifacts an important element in human conduct', pg. 444
The affordances provide organizations to use technological innovations and explore novel avenues. The avenues improve their internal factors such as transactional effectiveness, knowledge management, IT-enabled interactions, eventual information sharing, and so forth. To explore IT-enabled affordances, organizations' receptiveness to the environment and its openness to assimilate change does become important. Notwithstanding, external factors such as supply-push and demand-pull and interventionist approaches do influence the extent to which organizations assimilate change. Therefore, in this paper, the author examines the research question, 'In IT socio-technical system, for organizations to exhibit an open system what characteristics do they need to develop for radical product innovations to cumulate as stable designs.'

The composition of this paper is as follows. In the next section, the author discusses the theoretical perspective that provides a heuristic and analytic view to analyze organizations openness in assimilating changes and forms the basis of this study. The phenomena require examination in its setting, so the author selected a case study and discusses the details in section 3. In Section 4, the author discusses the organizational changes that are necessary for radical product innovations to cumulate as stable designs. Finally, the last section concludes the paper with the limitations of this research and directions for future research.

\section{Theoretical perspective}

Transition theories such as Multi-level Perspective (MLP) analyzed socio-technical transitions of steamships (Geels, 2002), aviation (Geels, 2006); Dutch nuclear energy (Geels \& Verhees, 2011), biogas (Raven \& Geels, 2010) to name a few. These studies were case study based and were done on successfully effected transitions. The MLP has three levels landscape, socio-technical regime, and nicheinnovation which are structurally separated and not hierarchically. Initially, it had two dimensions structural, temporal dimensions, later Raven et al., (2012) added spatial dimensions and provided MLP with necessary analytic and heuristic views to analyze sociotechnical transitions. In MLP, responses to external triggers at the landscape occur in strategic niche spaces, niche-innovation level, by radical innovations. The timing of the innovation decides the transition, referred to as the temporal dimension. The structuration levels at the regime and niche levels are physically separated and operate at different socio and economic levels. Apart from physical, there are also asymmetry, heterogeneity, and unevenness among actors, information and its flow, and knowledge levels. The physical, asymmetry, unevenness, and heterogeneity constitute the spatial dimension and influences interoperations among stakeholders. Despite the three dimensions, there is a need to arrive at a complete understanding of how the radical product innovations originating at niche-innovation level with interactions at the regime and niche levels cumulate as stable designs (Schot \& Geels, 2008). The responses to external triggers cause transition and based on the end-users wants-and-needs the triggers also decide the transition pathways (Kompella, 2017). Geels and Schot (2007) identified pathways or trajectories for socio-technical transitions, namely transformation, re-configuration, technological substitution, de-alignment and 
re-alignment, and reproduction. Based on the domain, for radical product innovations to cumulate as stable designs at the regime level any one or combination of the five pathways come into play. Government systems interact with society and researchers Kompella (2017) modified MLP as shown in Figure 1. The themes specific to government act at different levels of MLP; at the landscape are changing technologies, human elements, societal trends, and purpose and role of government while at regime are interaction and complexity and information management. The pathways and themes influence the way radical innovations cumulate as stable designs.

Figure 1. MLP framework for an IT related socio-technical system (E-Governance)

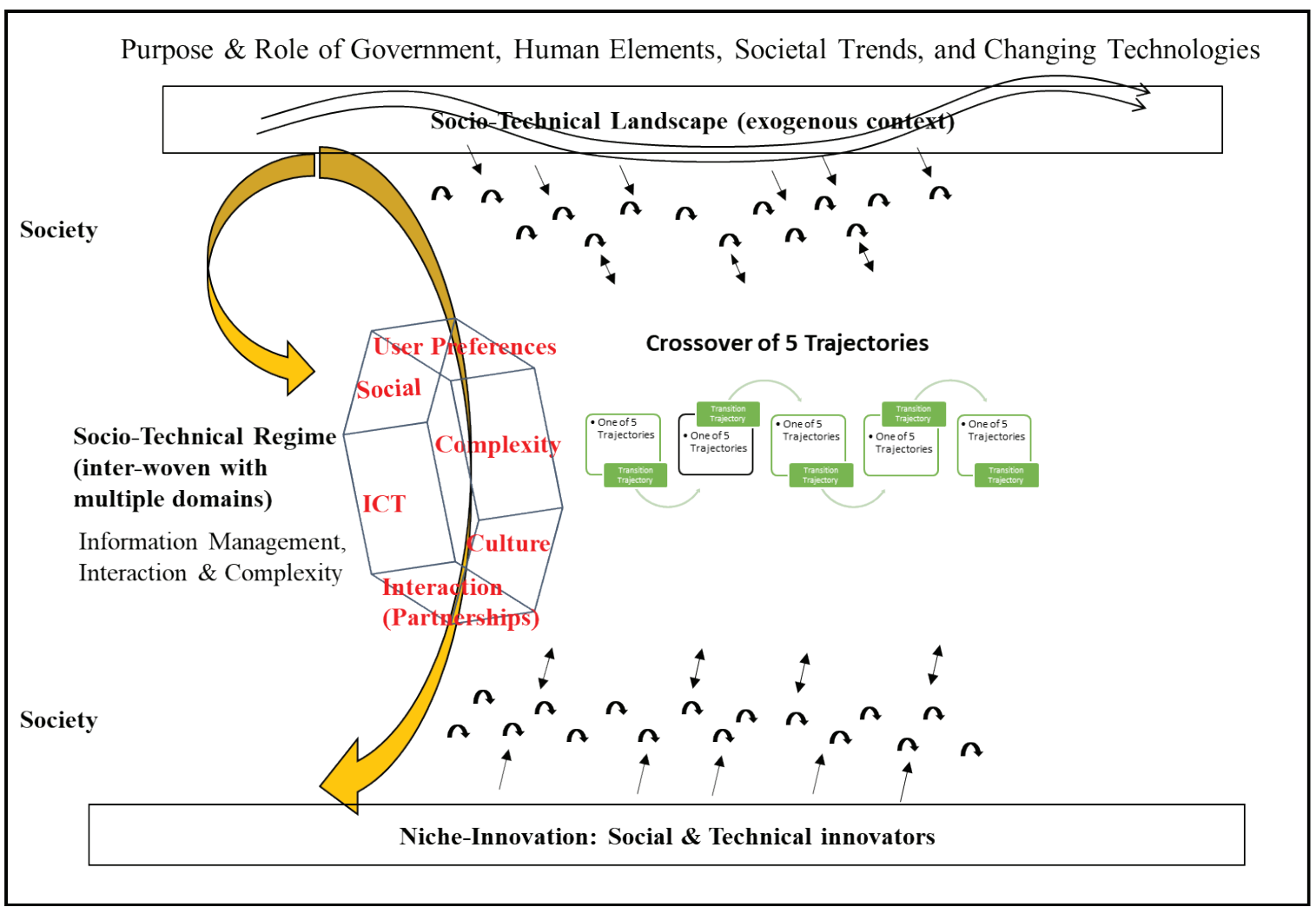

Source: Adapted from Kompella (2017)

Organizations and society influence the adoption of technology and the way they absorb innovation does vary. Organizations and society's evolution are heuristically and ontologically different (Witt, 2008). Organizations follow neo-Schumpeterian while society follows naturalistic evolutionary theory with marked differences in emergence, dissemination, and retention of novelty. To harness IT innovation benefits both entities need to co-evolve. Governments interact with society and for better responses to external triggers with suitable transition pathways along with cumulation of radical innovations they also need to assist the evolution of society. This requires modifications to configurations of the socio-technical system with appropriate changes to their rule-sets, thereby to their interoperations. Co-evolution of society is essential for the success of E-Governance, and organizations need to take the lead by providing affordances for society to IT innovations. In subsequent subsections of this chapter, the author discusses certain characteristics that organizations can consider for radical innovations to cumulate as stable designs.

\section{Open systems}

Open systems theory developed in the second half of the twentieth century refers to organizations' survival and its relationships with the environment. For open systems and other theories such as contingency theory, resources dependency, institutional theories encourage information to flow in and out, a source to set goals, explore alternatives and accomplish goals. Open systems tend to devise more than one way to accomplish goals or reach similar results with different conditions and operations, termed as equifinality (Bertalanffy, 1950). More than one way to accomplish goals (variation) leads to selecting from diverse alternatives (selection) and retaining the selected way (retention) when it assists in accomplishing the goal with optimal usage of resources. The variation-selection-retention in organizations leads to differentiation among their competitors leading to bigger market share. For sustainable strategies, it is necessary to include the environment with mutual interaction between organizations and entities in the environment. The process of reciprocal evolutionary change between two organisms that are ecologically dependent is co-evolution. 
Systems that support equifinality encourage alternatives and thereby competition. However, competition alone does not drive systems, humans cooperate with unrelated individuals and in large groups. Some economics refer to it as exogenous preferences and rational optimization. Apart from market forces and competition, cooperative exchanges and interactions would not be possible in self-interested individuals (Waring, 2010). Adoption of specific technological alternatives require cost advantage as compared to the existing technology; for example, cost savings are one of the most important motivations for customers to adopt cloud-based systems (Mital et al., 2015). Economic improvements are one of the reasons for radical innovations in the form of technological improvements and advancing functionalities to cumulate as stable designs. Slayton and Spinardi (2016), in their Boeing example of composite aircraft components, argue that different actors' views in the conceptualization of risk and their definition of conservative or radical innovations contrasts with several approaches to innovation studies which tend to adopt a structural view rather than a micro view. Therefore, for radical innovations to cumulate as stable designs apart from technological and economic considerations, the author includes necessary process improvements. The process improvements refer not only to technological innovations but also the integration of several organizational systems and their subsystems. The exploitation of economic and process improvements by the interdependent systems decides the acceptance, or cumulation, of radical innovations as stable designs.

Organizations create subsystems for reasons such as better management, information flow to and from outside and so forth. The broad classifications of the information flow from outside are economic, technological, socio-cultural and politico-legal. Organizations divide their subsystems in accordance with the four categories of information flow, namely personal (human resources management department), technical (R\&D or product development department), commercial (marketing, sales, purchase departments) and controller (controls information flow by providing right tools and each department provides feedback to improve customer services); these subsystems can also assist in providing avenues for co-evolution. The senior management controls all the four subsystems by providing direction by considering the environmental factors and decides the co-evolutionary characteristics of the organization and the other entities with which it interacts. McKelvey (1997) distinguished macro and micro co-evolution as intra- and inter-organizations respectively. Porter (2006) agrees with McKelvey (1997) co-evolution definition, but quotes Malerba (2006) statement and emphasizes the need to identify what is co-evolving with what and how intense this process and whether there is bidirectional of causality. This led to Porter (2006) develop co-evolutionary logic (or macro) and co-evolutionary mechanisms (micro). Porter (2006) identified six traits that define coevolutionary mechanisms and are specificity, reciprocity, simultaneity, genetic fixing, boundary crossing and organically derived. On the other hand, co-evolutionary logic provides sustainable strategies and the basic assumption that the firm has a symbiotic, co-evolving relationship with the greater society and ecosystem (Stead \& Stead, 2004) as quoted by Porter (2006). Therefore, for senior management to can utilize co-evolutionary logic to establish the organization's co-evolutionary logic by considering environmental factors that influence the organization and enable the organization's subsystems to develop the six micro co-evolutionary traits. The orchestrating involves senior management considering the external factors and establishing the co-evolutionary logic (macro) and enable organizations subsystems to display co-evolutionary mechanisms (micro). In the subsequent subsections of this section, the author further discusses co-evolution and policy formulation that can provide insights to orchestration by the senior management.

\section{Co-evolution}

In the biological sphere, species co-evolve to survive in their community while co-evolution in organizations increases their fitness to cope with the environment. The co-evolution driven by environmental changes does bring organizational changes. The scarcity of resources and competition comprise environmental changes. Organizations when going through changes can become isomorphic. The organization studies did explain these changes by considering selection, variation, and retention. On the other hand, strategic studies analyzed the organization's internal changes by examining managers capability to enhance fitness and survival or focused on adaptation. Both the studies independently analyzed adaptation and selection that occur in organizations. Organizations influence environments and organizational environments (consisting of other organizations) do get influenced by these environments. Therefore, a combined examination of adaptation-selection is necessary. Researchers across the world highlighted the need to go beyond organizational and strategic theories to address adaptation-selection. The work by Lewin and Volberda (1999) using co-evolution framework provides a new lens and directions to advance the adaptation-selection. Porter (2006) developed a co-evolution framework for organizations and the natural environment.

Porter (2006) identified three key points that make co-evolution frameworks unique and are 1) does not extend either strategy or organizational theories but makes new assumptions and models, 2) the human meaning-making process occurs more rapidly than biological co-evolution, and 3) the micro level (actor) activities measured by macro-level community effects (organizations interdependence, circular causality, iterative feedback and so forth).

The organization's co-evolution with other entities does define its intent and its actions; and based on external and internal factors can also become a constraint. The organizations selected in this paper are large monolithic systems with a large imprint. These organizations did ICT enable their procurement routines for a combination of reasons such as high-volume transactions, enhance transactional efficiency, procurement for and from multiple locations, and supply chains that involve essential commodities for diverse sections of the society. The managers with their learning process did perform the role of evangelizing and championing ICT initiatives. The learning process did enable them to perform their activities using ICT-enabled routines. 
The learning process referred to as the internal process, did form a coevolutionary logic with the external changes that involved both the organization's huge imprint and the other entities including society with which the organizations interact. The co-evolutionary logic did assist in improving transactional effectiveness but other aspects such as embedded agency did inhibit from taking further advantages of their ICT-enabled routines. The network collaboration that exists for these organizations also shaped their imprint and contributed to the inability of taking full advantage of their ICT-enabled routines.

The external changes include the inclusion of human elements such as privacy, trust, access, learning and so forth. Along with human elements, the reach of IT systems has also increased and is not confined to any select groups or communities. Therefore, societal trends such as digital divide, mobility, political participation, and enabling economic development of various sections do confront IT systems. These do influence the co-evolution of society and IT systems. For organizations need to consider the influence of external changes that include society and the interdependent organizations and consider co-evolution it is imperative to consider both co-evolutionary logic and micro co-evolution. shape the interactions between interoperation rule-sets. This necessitates the need for organizations to drive co-evolutionary logic and micro co-evolution traits and modify interoperations for radical innovations to cumulate as stable designs. Policy formulation can help organizations in achieving the co-evolutionary logic and micro co-evolution drive interoperation rule-sets, and, discussed in the next section.

\section{Policy formulation}

Selection of radical product innovation invariably requires actors at the regime level to adopt the innovation. The adoption requires certain adaptations or changes to the existing rule sets at regime with policy changes. Policy changes happen either top-down or using an embedded agency with changes occurring at a specific phase (end of the release). When changes occur at specific phases, the feedback from one phase feeds into the next product cycle - 'policy cycle' approach. 'Policy cycle' approach to account for refactoring the currently developed (released) system with feedback obtained at the end of the release. Reconciliation of these changes with existing product roadmap is necessary when changes are not aligned with current roadmap can result in a state of conflict, affecting the selection-adaptation cycle. Therefore, instead of at the end of the product cycle feedback is necessary at specific intervals from relevant stakeholders. The inclusion of stakeholders for feedback also plays a key role, by considering practices of people who implement the policy. This approach can help in developing a practice-oriented guide to policy instead of 'policy cycle' approach. Practice-oriented guide to policy assists in developing a policy that closely represents reality and does not deviate from reality (Colebatch, 2005).

Policy changes that impact the wider audience, say, citizens, may also require communication from policymakers to address social and ethical quandaries (Fedorowicz et al., 2010). A socio-technical system that has wider usage with several participating organizations getting involved at various stages, policy formulation to desist from following concomitant top-down policy formulation and involve embedded agency with inter-organizational collaboration. Organizations to use inter-organizational information system (IOS) for information sharing leading to shared decision-making. To achieve that organizations may also develop capabilities that allow them to coordinate with organizations (coordinative capability) and collaborate with wider stakeholders (combinative capability). The organization's procedures, systems, manuals, methods (system capability) to assist both coordinative and combinative capabilities.

Policies used in socio-technical systems define the business strategies of organizations that develop IT products. These organizations can assist in stabling designs of radical product innovations by using flexible and scalable product architecture that brings the required agility during product development. The developed product is operationalized by customizations/configurations to meet end-users wants-andneeds. For achieving the necessary agility, a unique combination of product development and operationalization (customizations/configurations) are necessary. The agility decides the extent to which radical product innovations stabilize as designs. The policy formulation to strive for and sustain agility. Organizations that develop IT products need to co-evolve with organizations that use their IT products. Both organizations, that develop and use IT products, need to adapt by making necessary changes to their organizational culture. Apart from product architectures influence process innovations, organizational culture does assist in the cumulation of radical product innovations as stable designs, the author has reserved this as future work and out-of-scope for this paper.

\section{Summary}

Transition theories such as MLP that are beyond the mere ontological representation of reality explain transitions using three structural levels and dimensions. It also explains the reasons for transitions and the levels at which triggers occur, the level at which response for trigger occurs, transition pathways and protected spaces for radical innovations and the location at which incremental innovations occur; however, complementing grand theory of MLP with local theories is essential for a complete understanding of radical innovations cumulating as stable designs (Geels \& Schot, 2007). The author identified three themes that assist in stabilization of radical innovations and are 1) organizations function as open systems 2) process innovations that influence and shape product architecture and organizational culture, and, 3) improvements to inter- and intra-organizational interoperations by improving organizational activities and capabilities. The focus of this paper is on the first theme.

For sustainability, organizations need to maintain a harmonious interaction with the environment. It is essential for organizations to focus on mutual benefit, at least in the long run, rather than exploiting their relationship for short-term gains. Therefore, organizations to have porous boundaries for receiving signals from the environment or focus their actions for developing an open system. As organizations focus on growth, for a mutual benefit, need to focus on the benefit 
of the environment by providing affordances and co-evolve with the environment. In Figure 2, the author depicts natural, built environment, society, and organization as concentric circles with the four organizational subsystems at the center of the concentric circles. The concentric circles also depict the hierarchical influence of environments on organizations. In the figure, dashed arrow lines depict macro co-evolution and solid arrow lines micro co-evolution.

Figure 2. Society and Organizations co-evolution with built and natural environments

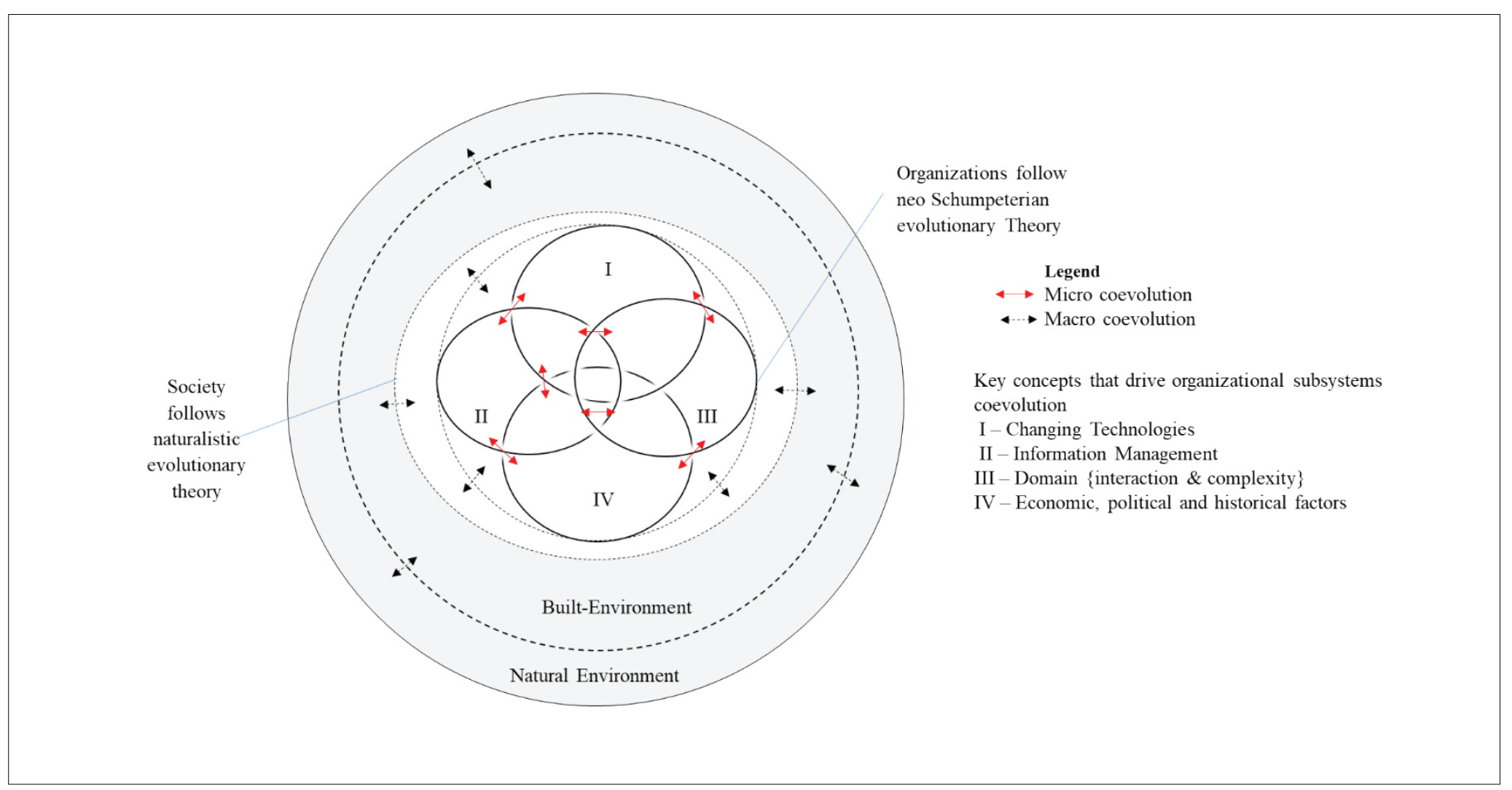

Source: Adapted from (Porter, 2006)

The forces that drive the organizational subsystems act at regime or landscape levels of the MLP. Organizational actors formulate policy to reflect co-evolutionary logic (macro co-evolution) and devise actions to represent any or a combination of the six micro co-evolutionary traits. Table 1 lists the six micro co-evolutionary traits, characteristics that require permanent and significant changes are in italics.

Table 1 Six characteristics of micro co-evolution

\begin{tabular}{l|l}
\hline Biological Characteristic & Organizational co-evolution \\
\hline Specificity & Local, sector-specific and path-dependent. \\
\hline Reciprocity & Changes to multiple organizational elements. \\
\hline Simultaneity & Change is mutual, in relation to the response of other elements. \\
\hline Genetic Fixing & Structural or other permanent forms of replication and identifiable. \\
\hline Boundary crossing & Involves two or more, unlike entities. Breakdown of cause-and-effect assumptions. \\
\hline Organically derived & Adaptive, emergent, and distinguished co-evolution from the intended or induced strategy - Self-organizing. \\
\hline
\end{tabular}

\{Source Co-evolution as research framework (Porter, 2006)\} 


\section{Case study methodology}

To better understand and analyze organizational dynamics in a sociotechnical system, it is essential to involve diverse organizations. In this paper, the author selected Central and state government organizations that IT-enabled their procurement activities by involving participating (either public or private) organizations at various stages of procurement. The selected organizations are large organizations that perform high-value procurement activities characterized by both volume and velocity. The organizational size did influence the demand-pull and supply-push in the procurement activities. The procurement occurred with deeply entrenched rule-sets shared by several organizations, and the size did shape these rule-sets. A singleminded focus on improving an internal factor of procurement activities, transactional effectiveness, and an interventionist approach did assist in shaping the rule-sets held by several organizations. An IT organization developed procurement software by considering the functions of the several organizations that participate in procurement; the software application did integrate procurement activities both by product (technical) and process innovations. The deeply entrenched rule-sets, policy, and operational based complexities did influence the integration activities. Selecting a single domain such as procurement does assist in identifying the policy commonalities, affordances that enable other entities, identifying situations for re-use of co-evolutionary traits across organizations (best practices), scenarios that inhibit display of coevolutionary traits, and so forth.

\section{Case selection and protocol}

Like several studies of transition, the author uses a case study and uses public E-Procurement in India. Public E-Procurement is a multi-faceted socio-technical system that involves information systems, public administration, supply chain, procurement, and policy. E-Procurement finds its application in the procurement of goods, especially where the volumes are large with varying frequencies. The regime involves several organizations with transitions caused by external triggers such as changing technologies, societal trends and human elements. For a multi-faceted system like E-Procurement involving several organizations and external triggers, the pathway or trajectory is transformation. Various industries, namely nuclear industry, oil refineries, warehouse and logistics, collieries, railways, forest produce, and so forth use E-Procurement not only as a marketplace for the purchase or sale of goods but also for the dispatch of goods by rail and/or road transports. In India, there is no legal framework governing public procurement, The Indian Contract Act 1872 and the Sales of Goods Act 1930 govern procurements (Nag, 2013). To capture the complexities and dynamics in Indian E-Procurement, the author used a multiple case study method (external validity) and selected cases from each of the industries with design following the guidelines mentioned in Yin (2009). Table 2 lists the selected cases along with their objectives and key characteristics. The cases required both public and participating organizations adhering or amending the rule-sets. The case selection considered three types of participating organizations that developed procurement software and managed the procurement activities, namely 1) Indian company with a global presence, 2) internal department and 3) a company that specializes in E-Procurement and performs all procurement activities with its own software. The software product in all the selected cases had product architecture that followed service-orientedarchitecture (SOA).

Fieldwork included primary sources from companies and government reports and records, written sources, mainstream media reports to source information. Thereby, construct validity. Using diverse methods, the author achieved data triangulation. The diverse information source helped in triangulating the identified constructs. One reason for relying on the primary source of data is a dearth of secondary literature on transitions from the perspective of the co-evolution of organizations as open systems. The author aimed output of inquiry at explaining specific implications that highlight the challenges at the regime level. Explanations also aimed at identifying scenarios that exhibited and challenges that inhibited co-evolutionary traits. For internal validity, the author used explanation building from the qualitative data and followed coding as suggested by Blair (2015). Interpretivism based on such an approach provides the required deductive processes in qualitative research (Hyde, 2000) and (Hossieni et al., 2012).

From the selected cases, it is discernibly evident that organizations perform public procurement as a sequence of steps. The department responsible for procurement analyzes the stock of material across the organization and performs data aggregation and sourcing solicitation strategies. The solicitation (or tender) involves arriving at procurement documents, evaluation criteria and prerequisites to qualify as sellers. Departments take the tender forward by advertising and conducting pre-bidding conferences to clarify any ambiguities and release Request for Proposals/Information/Quotations (RFX). Before proceeding with the tender process, prospective sellers who are eligible to participate in the selection process do pay a refundable deposit amount. IT system with the latest security mechanisms manages sellers' response to RFX including their bid price. Forward auctioning (sale of materials) or reverse auctioning (procurement) done using the IT system with the IT system rolling out the tender contract to the bid winner. 
Table 2 Summary of the selected cases

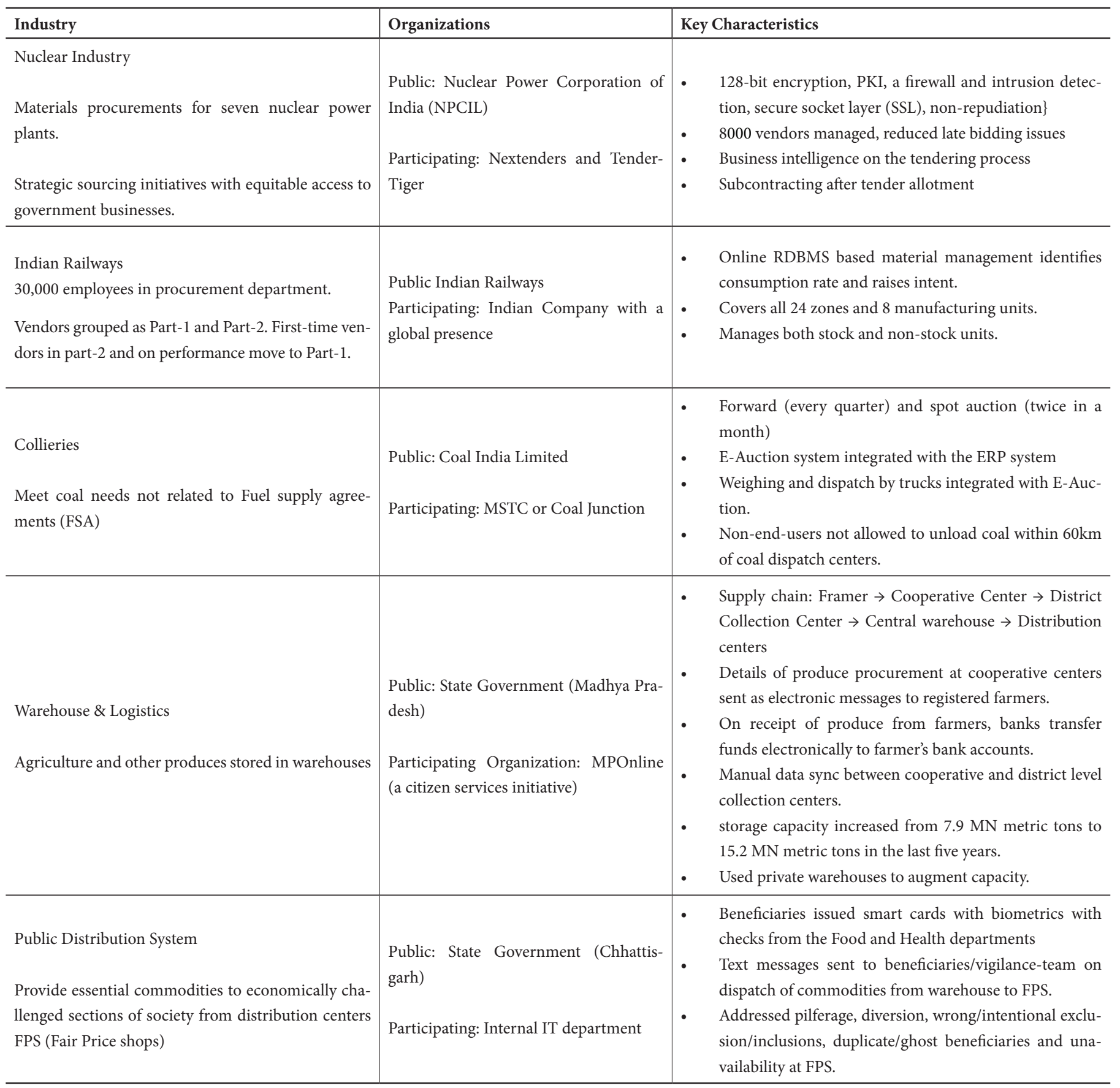

\section{Case synthesis}

From the information gathered from the selected cases, the author used coding, as suggested by Blair (2015), in three phases to synthesize the information. During coding, like in any coding method, it is necessary to follow a methodologically thoughtful approach and identify concepts, categories, and patterns and not use any pre-established tools. The concepts, categories, and patterns are later mapped to established theory, for this paper, the established theories are the transition and co-evolutionary theories. The author identified con- cepts, as suggested by Corbin and Strauss (1990), by coding concepts and not by lines-of-data. After identification of concepts, the author mapped the codes to categories and further to patterns; the author ensured that the categories/patterns are beyond grouping of concepts and represented certain properties and dimensions of the phenomenon. In the next step, the author used constructs from the established transition and co-evolutionary theory and mapped the patterns. Figure 3 and 4 summarizes the coding process followed by the author for both the actors who used and developed procurement software. 
Figure 3. The coding depicts the categories and the constructs from the transition and co-evolutionary theory - Actors who perform procurement activities

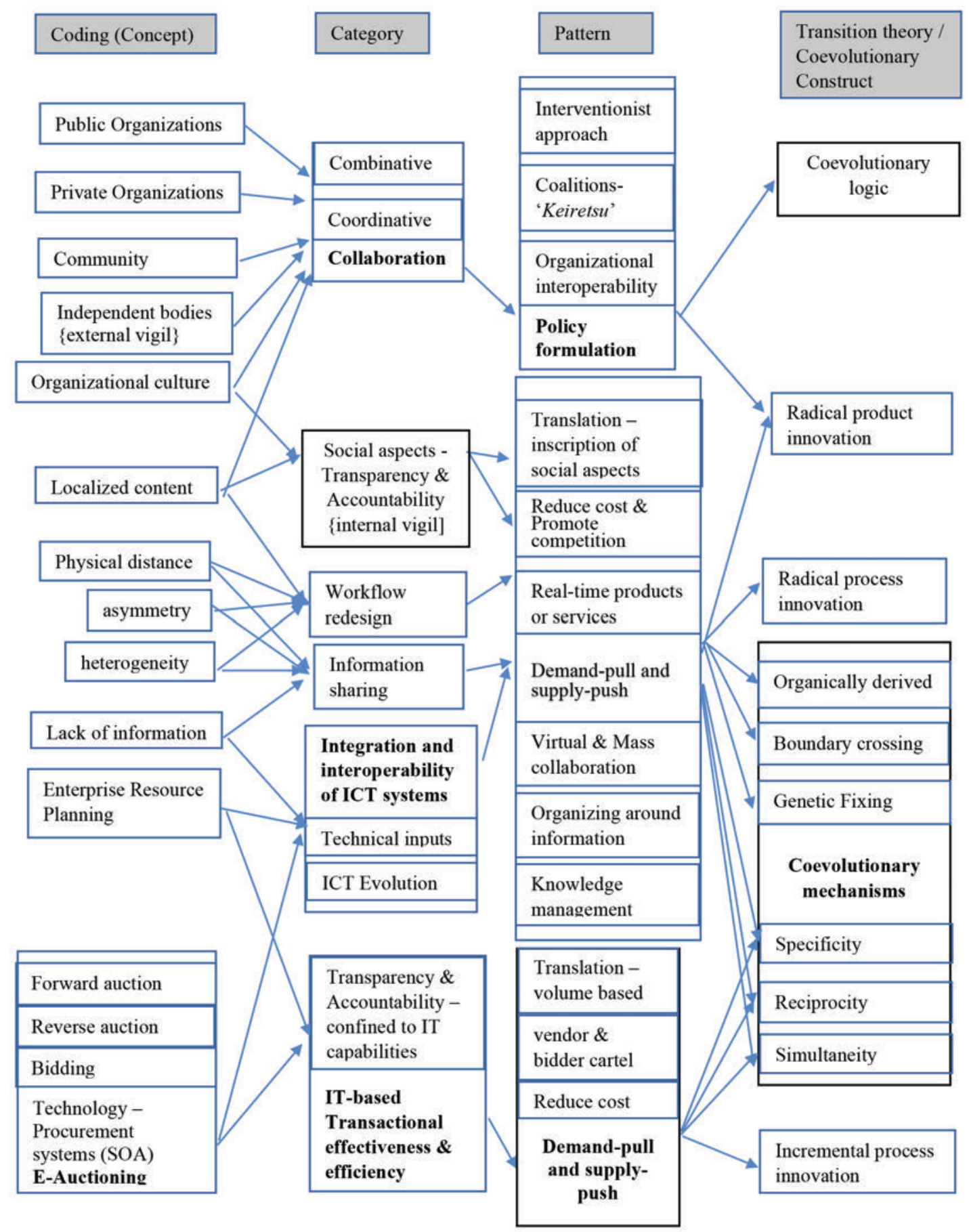


Figure 4. The coding depicts the categories and the constructs from the transition and co-evolutionary theory - Actors who develop procurement software

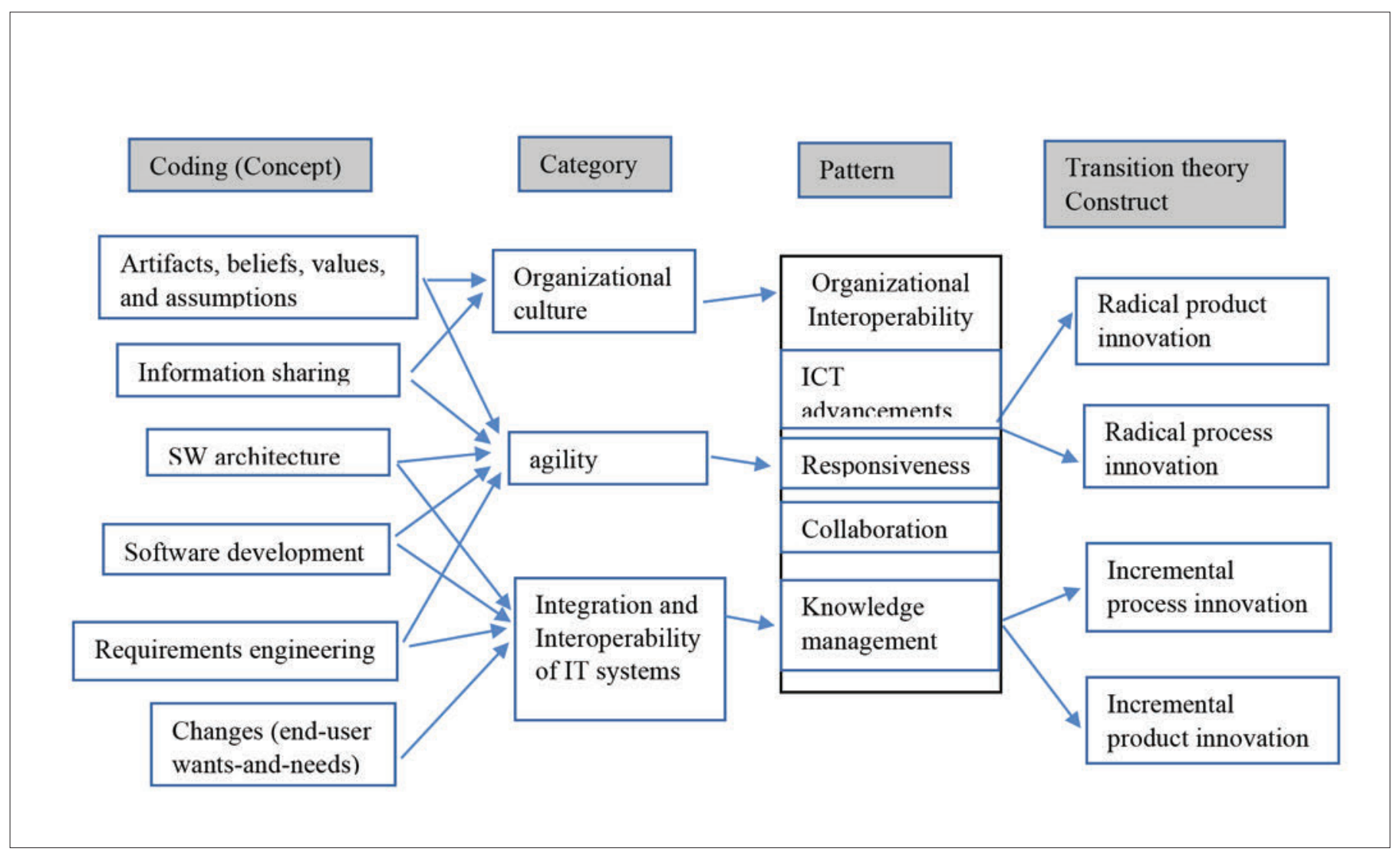

\section{Case findings}

The selected cases had the scale in terms of both the type and volume of materials and IT did assist in meeting scalability. In all the selected cases, the procurement software with its SOA architecture had the necessary memory, storage, and processing power to meet the desired scalability requirements. Each case did exhibit group formations and extended the embedded agency that existed in each of the cases. For example, the groups exhibited by cases that used participating organization (Nextenders, TenderTiger, MSTC or Coal Junction) that managed the procurement process was different from groups exhibited by Indian Railways or Public Distribution System. From the selected cases, the author noted two distinct areas that are worth discussing.

Technology. The volumes that existed in each case, a technologybased approach that assisted in meeting objectives and goals. The developments in SOA did resolve the spatial dimension, especially physical factor. Usage of technology to address other spatial factors were less limited. When public organizations outsourced procurement activities to participating organizations, especially managing the selection process, it did allow actors in public organization to focus on upstream activities of procurement. In other words, procurement objects such as forward and reverse auctioning were due to connections of the participating organization, albeit temporary. On the contrary, in cases Indian Railways and Public Distribution System, the public organization actors performed procurement objects and assigned procurement contracts to their select groups. However, the purpose of the E-Procurement in all the cases focussed on transaction effectiveness, therefore, if the interactions between the actors focussed on performing the activity as transaction oriented, network did perform its activities and the effect of selection activities either performed by the integrated or a separate group did not play a significant role. In some of the selected cases, the author noted no internet connectivity in cooperative centers with limited internet connectivity in district centers, organization locus of control did not extend over the entire length of the supply chain, change of transport logistics were left to the vendor to manage, no integrations with vendors' ERP systems, limited gathering of real-time data and/or automated systems to track, and stock or monitor of commodities at various stages. The feedback did exist and fed into policy formulation but a policy-cycle approach. Feedback does play a key role in evolving the system from its initial design constraints and a practice-oriented guide to policy can assist in better evolution of the system.

Local and Contingent forces. Vendors who can meet the volumes or have scope to scale their operations and meet volumes were preferred to get the contracts. The economies of scale do work to their advantage. Vendors did channel their efforts to establish 
themselves in the select vendor lists of the contracting organizations. Apart from quality, it requires making necessary specificity related changes. The changes were positioning delivery centers locally (geographically) close to public organization's consumption units. The vendors did utilize geographic, social, and political factors to assist them in their path dependency. The path dependency did become obligatory passage points. The forces (agency) from the vendor and the public organizations did drive the actors in the network to perform the ICT-enabled procurement activities, referred to as translation. Indeed, the translation required a contribution from both the vendor and public organization and included calculations, negotiations, persuasion, and some intrigue actions. The translations bring mobilization of resources, albeit, temporarily. In certain situations, translations did bring permanent mobilization of resources; actors formed groups, cartels, or family relationships or investments in vendor organizations and so forth (like keiretsu of Japanese governance); in other words, formed alliances. The new entrants had to work through these alliances and became obligatory passage points. The obligatory passage points did ensure the durability of translation and mobilization of resources, thereby providing sustainability.

In all the cases, the objectives of E-Procurement solution were transparency, efficiency, stimulate competition, reduce procurement cost (reduce time), larger vendor/bidder community and improve tracking and monitoring. The presence of obligatory passage points did address some of these objectives and caused certain irregularities. The irregularities were in the form of the award of contracts at exorbitant rates, acceptance of substandard supplies, failure to carry out quality checks, and manipulation at the tender processing stage to eliminate eligible bidders. Despite the award of punishments, we can attribute obligatory passage points to these irregularities. The properties of E-Procurement solution did enable the organizations to achieve transactional efficiency and address the physical aspect of the spatial dimension. The embeddedness of the agency did limit the influence of technology and reflected in the formation of alliances. In other words, the agency did not allow the technology to change its inscription about E-Procurement or the organization's influence with other entities.

The organizations continued with their existing design and did not attempt any changes to their policy, structure, and design; the actors did make policy changes to include changes for IT-based transactions. The actors did use feedback at the end of the cycle to update their methods and not an orchestrated feedback system that brings changes to organizational structure, policy, and design to continuously improve its adaptation. The influence of local and contingent factors was so significant that the actors viewed technology and its properties in a constrained view. The constrained view is the actor's realistic view of technology and the designs that did fit into their view cumulated as stable designs.

\section{Discussion}

Unlike other domains, IT is a fast-changing field. Actors in the selected cases used IT to transform the existing socio-technical system. The incumbent technology's inability to meet transaction volumes and the reach, IT could easily replace the incumbent technology. The organizations in the selected cases did exhibit considerable influence on the vendors mainly due to their size, volume, and the velocity. Apart from the organizational influence and the associated power and politics, the actors did form groups and assisted in influencing other vendor actors, or in other words did exhibit agency. These organizations being public organizations, the government's interventionist approach also assisted in actors exhibiting agency. The selected technology (IT) had the property to bridge physical distances and process huge amounts of data and addressed the need to connect geographically separated buyers and suppliers and the associated data volumes. The technology's properties can also become constraints and the author did notice that in the integration of the buyers and suppliers' IT systems. The constraints in integration did prevent from complete re-design of the workflow where the technology did confine to select sections of the workflow. In other words, the actors' discursive practices they had accumulated over a period and the artifacts of technology prevented them in re-design of the workflow. The actors' constructivist view of procurement activities did result in taking a deterministic view on the role that technology can play. Their approach was 'technology-deterministic' and confined it to their realistic view of technology.

The properties of technology and the procurement process get intertwined and become interrelated. The incumbent technology did shape the actors' constructivist view of procurement and their constructivist views got ingrained into the agency. The incumbent technology did play a transformational role until the actors' formed a constructivist view. Later, when the incumbent technology's properties became a constraint the actors resorted to substituting the incumbent technology with the IT technology. In doing so, actors intuitively followed a realistic view of IT technology (IT can address physical distances and process huge volumes of data - one of their realistic view) and followed re-configuration trajectory. The actors' view also reflected their anti-essentialism, i.e. properties of IT technology do not have any significance other than the properties that the actors believed that IT technology possessed. Instead, if the actors had taken a nonrealistic view of IT technology the actors view on the IT-enablement of procurement could have been in the path of anti-determinism. The anti-deterministic view can make the actors to explore transformational trajectory. A transformation trajectory better suits a public procurement system which are multi-faceted systems involving information systems, public administration, policy formulation, supplychain, and procurement. In the subsequent subsections, the author discusses the trajectory followed by the selected cases and the components that a transformational trajectory needs to consider for a coevolutionary framework.

\section{Transition trajectory of the selected cases}

The agency did assist the actors in using IT and redesigning the routines. As discussed earlier, they followed a realistic view of technology-deterministic approach. Actors used IT specifically during the source selection (tender) process and did bring transactional effectiveness and reduced procurement costs. To harness the advantages 
of IT, a multi-faceted system to achieve transparency, changes to the large vendor/bidder community, competitive bidding, changes in the process from procurement planning to contract management, and changes to the entire procurement supply chain by following a practice-oriented guide to policy. The agency in the multi-faceted system needs to go beyond the outcome that a realistic view of the deterministic approach provides, transactional effectiveness, a practice-oriented guide does assist in achieving that. From the selected cases, the author did note that actors followed a policy-cycle to account for IT enablement of routines. For a multi-faceted system involving diverse organizations and communities, it is necessary for a social construct of policy. In doing so, actors can include relevant social groups and their opinions and different meanings that result in diminishing interpretive flexibility, closure, and stabilization. The groups form networks that consist of actors; the actors have their interpretation of technology formulated policy. When actors merely extend the existing policy for IT-enabling of routines without any social construct of policy, actors do not consider co-evolutionary logic. Co-evolutionary logic can assist in connecting micro level changes (IT-enabled routines) to the strategic, sustainable, and societal goals that government organizations need to consider. In other words, actors did not define any co-evolutionary strategies for the mutual benefit of the society and organizations and attributable to the agency trained in an interventionist approach.
Along with policy formulation, changing actors view of technology properties and its relationship to social structures is necessary. A constructivism approach works for policy formulation, but to change the technology properties as suggested by Hutchby (2001) a conciliatory approach of affordances is suitable. The author from the selected cases did notice that the actors did visualize the entire workflow of the procurement process, thereby implemented the visualize the entire workflow affordance identified by Zammuto et al., (2007). In the procurement process, the other affordances identified by Zammuto et al., (2007), especially, mass and virtual collaboration can assist vendors/bidders and the buyers to collaborate and develop real-time products/services. The selected cases displayed only the procurement activities like tender, auction, and reverse auction that buyers (public organizations) wanted for their select products. Organizations activities assist them to perform micro co-evolutionary traits, where IT-enablement of procurement activities assist them in performing the specificity micro co-evolutionary trait. To achieve other micro co-evolutionary traits, organizations need to re-define their activities by combining affordances identified by Zammuto et al., (2007); the author lists these in Table 3.

Table 3. Affordances and related activities to enhance procurement

\begin{tabular}{|c|c|c|c|}
\hline Type of activity & Affordance [Theme] & Objective & Remarks \\
\hline \multirow[t]{2}{*}{ Product catalogs } & $\begin{array}{l}\text { Real-time products/service [Quick } \\
\text { search, classification, and so forth] }\end{array}$ & $\begin{array}{l}\text { Type of products procured and the role of the vendor. } \\
\text { Availability of detailed product specification - scope } \\
\text { for enhancements and improvements. }\end{array}$ & $\begin{array}{l}\text { Large vendor/buyer communities can } \\
\text { influence the availability of information }\end{array}$ \\
\hline & $\begin{array}{l}\text { Collaboration - mass/virtual [co- } \\
\text { llaboration for better product spe- } \\
\text { cifications] }\end{array}$ & Better quality products & $\begin{array}{l}\text { Local, contingent, and social context } \\
\text { can influence collaboration. }\end{array}$ \\
\hline $\begin{array}{l}\text { Integration of informa- } \\
\text { tion systems }\end{array}$ & $\begin{array}{l}\text { Visualize entire workflow [supply- } \\
\text { chain; shorter product delivery } \\
\text { times] }\end{array}$ & $\begin{array}{l}\text { Pluggable architecture that provides ease of service } \\
\text { provisioning, deployment, adaptation, integration, } \\
\text { operation, and exchange. }\end{array}$ & $\begin{array}{l}\text { It is essential to balance ease of ex- } \\
\text { change, deploy and adaptation with } \\
\text { integration. }\end{array}$ \\
\hline $\begin{array}{l}\text { Post procurement acti- } \\
\text { vity - maintenance and } \\
\text { service }\end{array}$ & $\begin{array}{l}\text { Collaboration }- \text { mass/virtual } \\
\text { [better knowledge management } \\
\text { towards improved operations] }\end{array}$ & Insights into post procurement activity & Transparency into the operational aspects \\
\hline $\begin{array}{l}\text { Post procurement activity } \\
\text { - transport }\end{array}$ & $\begin{array}{l}\text { Visualize entire workflow [supply- } \\
\text { chain; tracking of transport] }\end{array}$ & Efficient supply-chain & $\begin{array}{l}\text { Global Positioning System (GPS) ba- } \\
\text { sed tracking }\end{array}$ \\
\hline Organize & $\begin{array}{l}\text { Real-time products/services [or- } \\
\text { ganizations collaborate and share } \\
\text { information] }\end{array}$ & $\begin{array}{l}\text { Organize around information instead of tying infor- } \\
\text { mation to organizational structure }\end{array}$ & Requires significant changes at all levels. \\
\hline
\end{tabular}


Avgerou and Madon (2004) argue that organizational actions apart from considering local and contingent factors need to include social contexts. The constructivist view developed by actors on the procurement activities did include the local and contingent factors. With IT enablement of procurement activities and when organizations plan to expand their activities to achieve other co-evolutionary traits, their actions redefinition to include social contexts. The fifth affordance simulation representation between IT and organization can assist organizations in further refining their actions.

From the selected cases, the author did note that organizational actors did develop actions considering local and contingent factors and made necessary translations by bringing the agency into effect. The translations are towards IT-enablement of procurement activities and for improving transactional effectiveness and as envisaged by the interventionist approach. Along with the interventionist approach, the actor's constructivist view of procurement and their realistic view of technology decided their demand-pull and supply-push actions. These actions are a good initial starting point but unsuitable to manage transitions, cannot sustain strategic actions and do not enable other entities to co-evolve. Therefore, when organizations use IT innovations and aim to go beyond transactional effectiveness, it is necessary to identify activities (as listed in Table 3 ) and strive for system receptiveness with the outside environment. In doing so, both entities organizations and society need to co-evolve where the co-evolutionary logic enables entities to display the micro co-evolutionary traits. In the next section, the author explains micro co-evolutionary traits and their role in shaping the transformational trajectory.

\section{Co-evolutionary framework and transformational trajectory}

In the selected cases, the interventionist approach from Government of India did influence organizations policy decisions and other actions. The characteristic of the selected industries is their large partner ecosystem. The supply and demand pressures are so huge that these can assist in the co-evolution of organizations, despite their size and scale, and the partner ecosystems. Apart from the supply and demand pressures, the interventionist approach did influence the co-evolution of the organizations and the partner ecosystem. The co-evolutionary logic does shape the co-evolution of the organizations and partner ecosystems. The organizations did define their co-evolutionary logic, but the constructivist approach present in both organizations and the government (source of interventionist approach) decided their coevolutionary logic. As discussed earlier, the constructivist approach was technology deterministic and confined to their understanding of technology and its properties. For multi-faceted systems, there are diverse triggers that require responses that assist to cumulate radical innovations as stable designs. When actors perform routines with these stable designs, they can meet the diverse wants-and-needs of end-users. For continuously changing end-users wants-and-needs, a continuous generation of radical innovations and cumulate these as stable designs become necessary. In multi-faceted systems, like procurement, actors do develop constructivist views and these views can impede radical innovations and its cumulation as stable designs; therefore, while responding to transition with a trajectory, it becomes necessary to transition the constructivist views of actors to a conciliatory view on technology and its properties. In this regard, a transformational trajectory that brings changes at multiple levels better suits multi-faceted system like procurement.

For organizations to attempt changes, along with activities and related affordances listed in Table 3 it is essential for actors to consider the six micro co-evolutionary traits. These traits provide insights into other entities in the multi-faceted systems and their co-evolution. When organizations design these micro co-evolutionary traits into their system it assists in providing obligatory passage points. Apart from methods that assist in technology adoption, the design requires changes to both personal and organizational changes. In the rest of this section, the author discusses the changes, that assist transformational trajectory, in each of the six traits.

Specificity. Organizational routines do perform activities such as create, retrieve, store, and send information. The changes initiated by the organization are specific and confine to the local and contingent factors. In the selected cases, technology is the only visible change in the routines with no change to the constructivist view of actors. In certain selected cases, the local and contingent forces did drive technology adoption.

Reciprocity. Apart from the activities mentioned in specificity sharing of information does happen. Information sharing requires both inter-organizational interactions and information systems, IOS. The spatial dimension factors do come into play and require organizations to adjust their internal elements. The changes are towards developing capabilities such as system and absorptive.

Simultaneity. The organizations expand the capabilities developed in the trait reciprocity by making changes that correspond to other organizations.

As an initial starting point, organizations can attempt specificity, reciprocity, and simultaneity and address the spatial dimensional factors. When organizations address spatial dimension factors by using specificity, reciprocity, and spontaneity co-evolutionary traits it does result in agility. However, agility is a characteristic of the methods used to display the first three co-evolutionary traits. As mentioned by Russo and Fouts (1997), for co-evolution at the organizational level the top management commitment and intent are necessary and realize it at the middle and lower levels. To achieve changes at all the three levels the organizational culture does play a key role. As mentioned by Schein (2010), we can attempt organizational cultural changes at the three levels where artifacts, audios, and videos are at the first level, the employee beliefs, and values at the second, and the assumptions at the third level. To achieve other three micro co-evolutionary traits, changes to organizational culture become necessary.

Genetic fixing, Boundary crossing, and Organically derived. The co-evolutionary traits bring agility that is endogenous or emergent, unlike the agility that is characteristic of the method used. The author refers to this emergent as defined by Conboy (2007), 'readiness to 
rapidly or inherently create change, proactively or reactively embrace change and learn from change while contributing to perceived customer value through its collective components and relationships with its environment', pg. 340. While organizations strive for emergent agility it requires changes to both personal behavior and organizational structural changes.

\section{Conclusion and limitations}

Radical product innovations are responses that require actions from actors at multiple levels. Transition literature with stabilized transitions has highlighted the importance of radical product innovations but has fewer details regarding cumulation of radical product innovations. The author with procurement case studies did try to highlight the need for actions from multiple levels for radical innovations to cumulate as stable designs. Another characteristic of the selected cases is their interventionist approach. The stable designs are necessary to respond to external triggers; in multi-faceted systems, like procurement, the triggers are diverse and necessitates the need for co-evolution of other entities in the environment. More so, in an interventionist approach defining the co-evolutionary logic becomes important. In this paper, the author developed a framework for the co-evolution of organizations and the environment. The findings indicated that actors considered only local and contingent factors without any changes to co-evolutionary logic and with no focus on the co-evolution of entities in the environment. The approach did not emphasize shaping the engrained constructivist view of the actors. Such an approach does reflect in limited response to the external triggers where the organizations, in the selected cases, exhibited only the micro co-evolutionary trait specificity.

IT has become increasingly pervasive in everyday life and supplanted several coordination and control roles of the organizational hierarchy. For organizations to utilize the full benefit of IT, it is essential to explore new forms of organizing and make organizations an open system. Individuals and society are also adopting IT, combining IT with new forms of organizing organizations can also connect with society and the environment. By developing responsive and agile organizational systems co-evolution of both organizations and environment is possible. In this paper, selected cases did display one six micro co-evolutionary traits with no major changes to policy and macro co-evolutionary logic, by considering cases with more examples of co-evolution, it is possible to further expand the co-evolutionary framework.

\section{References}

Avgerou, C., \& Madon, S. (2004). Framing IS studies: understanding the social context of IS innovation (pp. 162-182). Oxford University Press, Oxford.

Blair, E. (2015). A reflexive exploration of two qualitative data coding techniques. Journal of Methods and Measurement in the Social Sciences, 6(1), 14-29.

Colebatch, H. K. (2005). Policy analysis, policy practice and political science. Australian Journal of Public Administration, 64(3), 14-23.
Conboy, K. (2009). Agility from first principles: Reconstructing the concept of agility in information systems development. Information systems research, 20(3), 329-354.

Corbin, J. M., \& Strauss, A. (1990). Grounded theory research: Procedures, canons, and evaluative criteria. Qualitative sociology, 13(1), 3-21.

Fedorowicz, J., Gogan, J. L., \& Culnan, M. J. (2010). Barriers to interorganizational information sharing in e-government: A stakeholder analysis. The Information Society, 26(5), 315-329.

Geels, F. W. (2002). Technological transitions as evolutionary reconfiguration processes: a multi-level perspective and a case-study. Research Policy, 31(8-9), 1257-1274.

Geels, F. W. (2006). Co-evolutionary and multi-level dynamics in transitions: the transformation of aviation systems and the shift from propeller to turbojet (1930-1970). Technovation, 26(9), 999-1016.

Geels, F. W., \& Schot, J. (2007). Typology of sociotechnical transition pathways. Research Policy, 36(3), 399-417.

Geels, F. W., \& Verhees, B. (2011). Cultural legitimacy and framing struggles in innovation journeys: a cultural-performative perspective and a case study of Dutch nuclear energy (1945-1986). Technological Forecasting and Social Change, 78(6), 910-930.

Hossieni, S., Dehkordi, G. J., \& Aghapour, H. A. (2012). Insights into case study: A discussion on forgotten aspects of case research. International Journal of Scientific and Research Publications, 2(3), 1-6.

Hutchby, I. (2001). Technologies, texts and affordances. Sociology, 35(2), 441-456.

Hyde, K. F. (2000). Recognizing deductive processes in qualitative research. Qualitative market research: An international journal, 3(2), 82-90.

Kompella, L. (2017). E-Governance systems as socio-technical transitions using multi-level perspective with case studies. Technological Forecasting and Social Change, 123, 80-94.

MacKenzie, D., \& Wajcman, J. (1999). Introductory essay: the social shaping of technology. The social shaping of technology, 2, 3-27.

Malerba, F. (2006). Innovation and the evolution of industries. Journal of Evolutionary Economics, 16, 3-23.

McKelvey, B. (1997). Quasi-natural organization science. Organization Science, 8(4), 352-380.

Mital, M., Pani, A. K., Damodaran, S., \& Ramesh, R. (2015). Cloud based management and control system for smart communities: A practical case study. Computers in Industry, 74, 162-172.

Nag, B. (2013). Combating Corruption in Public Procurement-A Case Study of the Indian Railways. 
Porter, T. B. (2006). Coevolution as a research framework for organizations and the natural environment. Organization \& Environment, 19(4), 479-504.

Raven, R. P. J. M., \& Geels, F. W. (2010). Socio-cognitive evolution in niche development: Comparative analysis of biogas development in Denmark and the Netherlands (1973-2004). Technovation, 30(2), 87-99.

Raven, R., Schot, J., \& Berkhout, F. (2012). Space and scale in sociotechnical transitions. Environmental Innovation and Societal Transitions, 4, 63-78.

Russo, M. V., \& Fouts, P. A. (1997). A resource-based perspective on corporate environmental performance and profitability. Academy of management Journal, 40(3), 534-559.

Schein, E. H. (2010). Organizational culture and leadership (Vol. 2). John Wiley \& Sons.

Schot, J., \& Geels, F. W. (2008). Strategic niche management and sustainable innovation journeys: theory, findings, research agenda, and policy. Technology analysis \& strategic management, 20(5), 537-554.
Slayton, R., \& Spinardi, G. (2016). Radical innovation in scaling up: Boeing's Dreamliner and the challenge of socio-technical transitions. Technovation, 47, 47-58.

Von Bertalanffy, L. (1950). The theory of open systems in physics and biology. Science, 111(2872), 23-29.

Waring, T. M. (2010). New evolutionary foundations: Theoretical requirements for a science of sustainability. Ecological Economics, 69(4), 718-730.

Witt, U. (2008). What is specific about evolutionary economics? Journal of Evolutionary Economics, 18(5), 547-575.

Yin, R. K. (2009). Case study research: Design and methods. Sage publications.

Zammuto, R. F., Griffith, T. L., Majchrzak, A., Dougherty, D. J., \& Faraj, S. (2007). Information technology and the changing fabric of organization. Organization science, 18(5), 749-762. 